\title{
Correction to: Compact Perturbations of a Strongly Positive Operator
}

\author{
J. M. Soriano® and M. Ordoñez Cabrera
}

\section{Correction to: Mediterr. J. Math. (2018) 15:23 https://doi.org/10.1007/s00009-018-1069-0}

Professor Biagio Ricceri recently sent us a counterexample to a result in our original paper. After a revision of the paper, we have detected a non-correct use of Sard-Smale theorem in the proof of Section (aI-2-2) of Theorem 7. This leads us to add hypothesis (iv) to this theorem. In the particular case in which the operator $B$ is linear, the hypothesis (iv) coincides with (iii) and it is non superfluous.

We keep all the notations in the original paper.

Theorem 7 now runs as follows:

Theorem 7. Let $A, B: X \rightarrow X^{*}$ be two operators, where $X$ is a real Hilbert space and $X^{*}$ its dual space.

We assume that:

(i) $A$ is a linear continuous strongly positive operator.

(ii) $B$ is a $C^{1}$-compact operator.

(iii) The operator family $\{A+t B\}_{t \in[0,1]}$ is uniformly weakly coercive, i.e, $\|u\| \rightarrow \infty$ if and only if $\inf _{t \in[0,1]}\|(A+t B) u\| \rightarrow \infty$

(iv) The operator family $\left\{A+t B^{\prime}(v)\right\}_{t \in[0,1]}$ is uniformly weakly coercive for all $v \in X$.

Then the following assertions hold:

(a) The equation $(A+B)(u)=b$ has at least one solution and at most finitely many solutions for each fixed $b \in X^{*}$.

The original article can be found online at https://doi.org/10.1007/s00009-018-1069-0. 
(b) In the particular case in which $B$ is a linear compact operator, the equation $(A+B)(u)=b$ has one and only one solution.

Proof. We only modify the proof of sections (aI-2-2) and (b).

(aI-2-2) We will prove here that each $b \in X^{*}$ is a regular value of the operator $A+t B$, where $t$ is a fixed $t \in[0,1]$, and that $A+t B^{\prime}(v) \in$ $\operatorname{Isom}\left(X, X^{*}\right)$ for any $t \in[0,1]$.

Since for any fixed $t \in[0,1]$, and fixed $v \in X, t B^{\prime}(v) \in \mathcal{L}\left(X, X^{*}\right)$ is a compact operator (Theorem 3), Theorem 2 implies that $A+t B^{\prime}(v) \in$ $\mathcal{F}\left(X, X^{*}\right)$ and

$$
\operatorname{Ind}\left(A+t B^{\prime}(v)\right)=\operatorname{Ind}(A)=0,
$$

for fixed $t \in[0,1]$ and any fixed $v \in X$.

Since $A+t B^{\prime}(v)$ is weakly coercive, we obtain that $\operatorname{ker}\left(A+t B^{\prime}(v)\right)=$ $\{0\}$. Indeed, if were not the case, $\operatorname{ker}\left(A+t B^{\prime}\left(v_{0}\right)\right) \neq\{0\}$ for some $v_{0}$, we would take $x \in \operatorname{ker}\left(A+t B^{\prime}\left(v_{0}\right)\right) \backslash\{0\}$. Let $\lambda \in(0,+\infty)$, then

$$
\|\lambda x\|=|\lambda|\|x\| \rightarrow \infty \text { when } \lambda \rightarrow \infty .
$$

Therefore

$$
\left\|\left(A+t B^{\prime}\left(v_{0}\right)\right)(\lambda x)\right\|=|\lambda|\left\|\left(A+t B^{\prime}\left(v_{0}\right)\right)(x)\right\| \rightarrow \infty \quad \text { as } \lambda \rightarrow \infty .
$$

However

$$
\left(A+t B^{\prime}\left(v_{0}\right)\right)(\lambda x)=\lambda\left(A+t B^{\prime}\left(v_{0}\right)\right)(x)=\lambda .0=0,
$$

because of being $x \in \operatorname{ker}\left(A+t B^{\prime}\left(v_{0}\right)\right)$. This is a contradiction, therefore $\operatorname{ker}\left(A+t B^{\prime}(v)\right)=\{0\}$ for all $v \in X$.

Since $\operatorname{Ind}\left(A+t B^{\prime}(v)\right)=0$, and $\operatorname{dim}\left(\operatorname{ker}\left(A+t B^{\prime}(v)\right)=0\right.$, we have $\operatorname{codim}\left(\mathrm{R}\left(A+t B^{\prime}(v)\right)\right)=0$. Hence $A+t B^{\prime}(v)$ is surjective, and $A+t B^{\prime}(v)$ $\in \operatorname{Isom}\left(X, X^{*}\right)$.

Since $A=A^{\prime}(v), \quad A+t B^{\prime}(v)=A^{\prime}(v)+t B^{\prime}(v)=(A+t B)^{\prime}(v)$, then $(A+t B)^{\prime}(v)$ is surjective for all $v \in X$.

Let $b \in X^{*}$, then $(A+t B)^{-1}(b)=\emptyset$ or there is $v \in X$ such that $(A+t B)(v)=b$. Since $(A+t B)^{\prime}(v)$ is surjective, $b$ is a regular value of the operator $A+t B$.

(b) Since the number of solutions of $(A+B)(u)=b$ is finite and $A+B$ is a linear operator, the number of solutions is one and only one because if there were more than one, all convex combinations of these would also be solutions, and then the number would be infinite

Therefore Corollary 8 is established as follows:

Corollary 8. Let $A, B: X \rightarrow X$ be two operators, where $X$ is a real Hilbert space.

We assume that:

(i) $A$ is a linear continuous strongly positive operator.

(ii) $B$ is a $C^{1}$-compact operator.

(iii) The operator family $\{A+t B\}_{t \in[0,1]}$ is uniformly weakly coercive.

(iv) The operator family $\left\{A+t B^{\prime}(v)\right\}_{t \in[0,1]}$ is uniformly weakly coercive for all $v \in X$.: 
(a) The equation $(A+B)(u)=b$ has at least one solution and at most finitely many solutions for each fixed $b \in X$.

(b) In the particular case in which $B$ is a linear compact operator, the equation $(A+B)(u)=b$ has one and only one solution.

\section{Examples}

Let us first observe that in the given examples, $B$ is a linear compact operator, then hypothesis (iii) coincides with hypothesis (iv). Therefore we have to give sufficient conditions so that hypothesis (iii) is satisfied.

Example 1. It is shown in [1] that $\|B u\| \leq\|k\|\|u\|$. Assume that $c-\|k\|>0$.

Since $\|B u\| \leq\|k\|\|u\|$ and $c-\|k\|>0$, we have

$$
\begin{aligned}
(\|A\|+\|B\|)\|u\| & \geq\|A u+t B u\| \geq\|A u\|-t\|B u\| \\
& >(c-t\|k\|)\|u\| \geq(c-\|k\|)\|u\|,
\end{aligned}
$$

which implies the uniform weakly coerciveness of the family $\{A+t B\}_{t \in[0,1]}$. Example 2. Since $A$ is the duality map from $X_{E}$ to $X_{E}^{*}$,

$$
\|A u\|_{X_{E}^{*}}=\|u\|_{X_{E}}=\|u\|_{1,2} .
$$

Since $j: X \rightarrow X_{E}^{*}$ is a injective linear continuous operator, there is $k>0$ such that

$$
\|B u\|_{X_{E}^{*}}=\|j \circ F(u)\|_{X_{E}^{*}} \leq k\|F u\| .
$$

Since $\|\cdot\|_{1,2}$ and $\|\cdot\|$ are equivalent norms, we can write

$$
\|F u\| \leq c\|F u\|_{1,2} .
$$

Therefore

$$
\|B u\|_{X_{E}^{*}} \leq c k\left[m(G)^{\frac{1}{2}}\|f\|_{1,2}\right]\|u\|_{1,2} .
$$

Assume that $c k\left[m(G)^{\frac{1}{2}}\|f\|_{1,2}\right]<1$. We have

$$
\begin{aligned}
(\|A\|+\|B\|)\|u\|_{1,2} \geq\|A u+t B u\|_{X_{E}^{*}} & \geq\left|\|A u\|_{X_{E}^{*}}-t\|B u\|_{X_{E}^{*}}\right| \\
& >\left(1-t c k\left[m(G)^{\frac{1}{2}}\|f\|_{1,2}\right]\right)\|u\|_{1,2} \\
& \geq\left(1-c k\left[m(G)^{\frac{1}{2}}\|f\|_{1,2}\right]\right)\|u\|_{1,2},
\end{aligned}
$$

which implies the uniform weakly coerciveness of the family $\{A+t B\}_{t \in[0,1]}$.

\section{Acknowledgements}

We are very grateful to Professor Biagio Ricceri for providing a counterexample which led us to revise the original version of the paper. 


\section{Reference}

[1] Zeidler, E.: Nonlinear functional analysis and its applications IIA. Springer, New York (1992)

J. M. Soriano and M. Ordoñez Cabrera

Departamento de Análisis Matemático, Facultad de Matemáticas

Universidad de Sevilla

Avda. Reina Mercedes s/n

41012 Seville

Spain

e-mail: soriano@us.es

M. Ordoñez Cabrera

e-mail: cabrera@us.es 